PROCEEDINGS OF THE

AMERICAN MATHEMATICAL SOCIETY

Volume 135, Number 1, January 2007, Pages 129-134

S 0002-9939(06)08553-4

Article electronically published on June 19, 2006

\title{
TWO ENTRIES ON BILATERAL HYPERGEOMETRIC SERIES IN RAMANUJAN'S LOST NOTEBOOK
}

\author{
BRUCE C. BERNDT AND WENCHANG CHU
}

(Communicated by Jonathan M. Borwein)

\begin{abstract}
Two entries of bilateral sums in Ramanujan's lost notebook are confirmed by means of Dougall's bilateral series identities with one of them being corrected.
\end{abstract}

\section{INTRODUCTION}

On page 200 of his lost notebook, in the pagination of [4], Ramanujan offers two results on certain bilateral hypergeometric series. As we shall see, the second follows from a theorem of J. Dougall [2]. The first gives a formula for the derivative of a quotient of two certain bilateral hypergeometric series. Ramanujan's formula needs to be slightly corrected, but what is remarkable is that such a formula exists! This is one of those instances where we can undauntedly claim that if Ramanujan had not discovered the formula, no one else, at least in the foreseeable future, would have done so.

We first state the second formula, which requires modest deciphering, because of Ramanujan's use of ellipses to denote missing terms. It will be used in the proof of Ramanujan's first formula on page 200.

Entry 1.1 (p. 200). Let $\alpha, \beta, \gamma, \delta$, and $\xi$ be complex numbers such that $\operatorname{Re}(\alpha+\beta+\gamma+\delta)>3$. Then

$$
\begin{aligned}
& \sum_{n=-\infty}^{\infty} \frac{\xi+2 n}{\Gamma(\alpha+\xi+n) \Gamma(\beta-\xi-n) \Gamma(\gamma+\xi+n) \Gamma(\delta-\xi-n) \Gamma(\alpha-n) \Gamma(\beta+n) \Gamma(\gamma-n) \Gamma(\delta+n)} \\
& =\frac{\sin (\pi \xi) \Gamma(\alpha+\beta+\gamma+\delta-3)}{\pi \Gamma(\alpha+\gamma+\xi-1) \Gamma(\beta+\delta-\xi-1) \Gamma(\alpha+\beta-1) \Gamma(\beta+\gamma-1) \Gamma(\gamma+\delta-1) \Gamma(\delta+\alpha-1)} .
\end{aligned}
$$

We state a corrected version of Ramanujan's more interesting formula. At the end of the paper, we indicate the mistakes in Ramanujan's original formula.

Entry 1.2 (Corrected, p. 200). Define, for any real numbers $\theta$ and $s$ and for any complex numbers $\alpha, \beta, \gamma$, and $\delta$, such that $\operatorname{Re}(\alpha+\beta+\gamma+\delta)>4$,

$$
\varphi_{s}(\theta):=\sum_{n=-\infty}^{\infty} \frac{e^{(n+s) i \theta}}{\Gamma(\alpha+s+n) \Gamma(\beta-s-n) \Gamma(\gamma+s+n) \Gamma(\delta-s-n)} .
$$

Received by the editors July 23, 2005.

2000 Mathematics Subject Classification. Primary 33C20.

The first author's research was partially supported by grant MDA904-00-1-0015 from the National Security Agency. 
Then

$$
\frac{d}{d \theta} \frac{\varphi_{s}(\theta)}{\varphi_{t}(\theta)}=\frac{i \sin \{\pi(s-t)\}\left(2 \sin \frac{\theta}{2}\right)^{\alpha+\beta+\gamma+\delta-4} e^{i(\pi-\theta)(\alpha-\beta+\gamma-\delta+2 s+2 t) / 2}}{\pi \varphi_{t}^{2}(\theta) \Gamma(\alpha+\beta-1) \Gamma(\beta+\gamma-1) \Gamma(\gamma+\delta-1) \Gamma(\delta+\alpha-1)}
$$

\section{BACKGRound ON BILATERAL SERIES}

For every integer $n$, define

$$
(a)_{n}:=\frac{\Gamma(a+n)}{\Gamma(a)} .
$$

The bilateral hypergeometric series ${ }_{p} H_{p}$ is defined for complex parameters $a_{1}, a_{2}, \ldots$, $a_{p}$ and $b_{1}, b_{2}, \ldots, b_{p}$ by

$$
{ }_{p} H_{p}\left[\begin{array}{l}
a_{1}, a_{2}, \ldots, a_{p} ; \\
b_{1}, b_{2}, \ldots, b_{p} ;
\end{array}\right]:=\sum_{n=-\infty}^{\infty} \frac{\left(a_{1}\right)_{n}\left(a_{2}\right)_{n} \cdots\left(a_{p}\right)_{n}}{\left(b_{1}\right)_{n}\left(b_{2}\right)_{n} \cdots\left(b_{p}\right)_{n}} z^{n} .
$$

With the use of D'Alembert's ratio test, it can be checked that ${ }_{p} H_{p}$ converges only for $|z|=1$, provided that [5, p. 181, eq. (6.1.1.6)]

$$
\operatorname{Re}\left(b_{1}+b_{2}+\cdots+b_{p}-a_{1}-a_{2}-\cdots-a_{p}\right)>1
$$

The series ${ }_{p} H_{p}$ is said to be well poised if

$$
a_{1}+b_{1}=a_{2}+b_{2}=\cdots=a_{p}+b_{p} .
$$

In 1907, J. Dougall 2] proved that a well-poised series ${ }_{5} H_{5}$ could be evaluated at $z=1$. In order to state this evaluation, define

$$
\Gamma\left[\begin{array}{c}
a_{1}, a_{2}, \ldots, a_{m} \\
\beta_{1}, b_{2}, \ldots, b_{n}
\end{array}\right]:=\frac{\Gamma\left(a_{1}\right) \Gamma\left(a_{2}\right) \cdots \Gamma\left(a_{m}\right)}{\Gamma\left(b_{1}\right) \Gamma\left(b_{2}\right) \cdots \Gamma\left(b_{n}\right)} .
$$

Then Dougall's formula [5, p. 182, eq. (6.1.2.5)] is given by

$$
\begin{aligned}
& { }_{5} H_{5}\left[\begin{array}{ccccr}
1+\frac{1}{2} a, & b, & c, & d, & e ; \\
\frac{1}{2} a, & 1+a-b, & 1+a-c, & 1+a-d, & 1+a-e ;
\end{array}\right] \\
& =\Gamma\left[\begin{array}{l}
1-b, 1-c, 1-d, 1-e, 1+a-b, 1+a-c, 1+a-d, \\
1+a, 1-a, 1+a-b-c, 1+a-b-d, 1+a-b-e,
\end{array}\right. \\
& \left.\begin{array}{l}
1+a-e, 1+2 a-b-c-d-e \\
1+a-c-d, 1+a-c-e, 1+a-d-e
\end{array}\right],
\end{aligned}
$$

where, for convergence, by (2.2),

$$
1+\operatorname{Re}(2 a-b-c-d-e)>0 .
$$

We need one further result, namely, the bilateral binomial theorem discovered by M. E. Horn [3]. If $a$ and $c$ are complex numbers $\operatorname{Re}(c-a)>1$ and $z$ is a complex number with $|z|=1$, then

$$
{ }_{1} H_{1}\left[\begin{array}{l}
a ; \\
c ;
\end{array}\right]=\frac{(1-z)^{c-a-1}}{(-z)^{c-1}} \frac{\Gamma(1-a) \Gamma(c)}{\Gamma(c-a)} .
$$

It would seem that Ramanujan had discovered (2.5), but we are unaware of any mention of it by Ramanujan in his papers or notebooks. We remark that the bilateral binomial theorem can also be recovered from another bilateral hypergeometric series identity [1, p. 110, Thm. 2.8.2] due to Dougall [2], namely,

$$
{ }_{2} H_{2}\left[\begin{array}{ll}
a, & b ; \\
c, & d ;
\end{array}\right]=\frac{\Gamma(1-a) \Gamma(1-b) \Gamma(c) \Gamma(d) \Gamma(c+d-a-b-1)}{\Gamma(c-a) \Gamma(c-b) \Gamma(d-a) \Gamma(d-b)},
$$


where $\operatorname{Re}(c+d-a-b)>1$ for convergence. In fact, in the identity above, first replacing $b$ by $d z$ and second letting $d \rightarrow+\infty$, we derive (2.5) in view of Stirling's asymptotic formula for the $\Gamma$-function.

In the sequel, we very often use the classical reciprocal formula

$$
\Gamma(z) \Gamma(1-z)=\frac{\pi}{\sin (\pi z)} .
$$

\section{Proof of Entry 1.1}

We show that (2.3) leads to a proof of Entry 1.1 .

Proof. Let $S$ denote the series on the left-hand side of (1.1). Define

$$
\Omega:=\frac{\sin \{\pi(\beta-\xi)\} \sin \{\pi(\delta-\xi)\} \sin \{\pi \alpha\} \sin \{\pi \gamma\}}{\pi^{4}} .
$$

Using (2.7) and (3.1), we see that we can write $S$ in the form

$$
\begin{aligned}
S & =\Omega \xi \sum_{n=-\infty}^{\infty} \frac{(\xi+2 n) \Gamma(1+\xi+n-\beta) \Gamma(1+\xi+n-\delta) \Gamma(1+n-\alpha) \Gamma(1+n-\gamma)}{\xi \Gamma(\alpha+\xi+n) \Gamma(\gamma+\xi+n) \Gamma(\beta+n) \Gamma(\delta+n)} \\
& =\Omega \xi \frac{\Gamma(1+\xi-\beta) \Gamma(1+\xi-\delta) \Gamma(1-\alpha) \Gamma(1-\gamma)}{\Gamma(\alpha+\xi) \Gamma(\gamma+\xi) \Gamma(\beta) \Gamma(\delta)}
\end{aligned}
$$

$$
\times \sum_{n=-\infty}^{\infty} \frac{\left(1+\frac{1}{2} \xi\right)_{n}(1-\alpha)_{n}(1+\xi-\beta)_{n}(1-\gamma)_{n}(1+\xi-\delta)_{n}}{\left(\frac{1}{2} \xi\right)_{n}(\alpha+\xi)_{n}(\beta)_{n}(\gamma+\xi)_{n}(\delta)_{n}}
$$

Note that the series (3.2) is well poised, and so we can invoke (2.3) with $a=\xi$, $b=1-\alpha, c=1+\xi-\beta, d=1-\gamma$, and $e=1+\xi-\delta$. Thus, for $\operatorname{Re}(\alpha+\beta+\gamma+\delta)>3$ for convergence, we deduce that

$$
\begin{aligned}
S= & \Omega \xi \frac{\Gamma(1-\alpha) \Gamma(1+\xi-\beta) \Gamma(1-\gamma) \Gamma(1+\xi-\delta)}{\Gamma(\alpha+\xi) \Gamma(\beta) \Gamma(\gamma+\xi) \Gamma(\delta)} \times \frac{\Gamma(\alpha) \Gamma(\beta) \Gamma(\gamma) \Gamma(\delta)}{\Gamma(\alpha+\gamma+\xi-1) \Gamma(\beta+\delta-\xi-1)} \\
& \times \frac{\Gamma(\alpha+\xi) \Gamma(\beta-\xi) \Gamma(\gamma+\xi) \Gamma(\delta-\xi) \Gamma(\alpha+\beta+\gamma+\delta-3)}{\Gamma(1+\xi) \Gamma(1-\xi) \Gamma(\alpha+\beta-1) \Gamma(\beta+\gamma-1) \Gamma(\gamma+\delta-1) \Gamma(\delta+\alpha-1)} \\
= & \frac{\sin (\pi \xi) \Gamma(\alpha+\beta+\gamma+\delta-3)}{\pi \Gamma(\alpha+\gamma+\xi-1) \Gamma(\beta+\delta-\xi-1) \Gamma(\alpha+\beta-1) \Gamma(\beta+\gamma-1) \Gamma(\gamma+\delta-1) \Gamma(\delta+\alpha-1)}
\end{aligned}
$$

where we applied (2.7) five times, used the value of $\Omega$ from (3.1), and simplified.

\section{Proof of Entry 1.2}

We first replace the functions in Entry 1.2 by another pair with which it is easier to work. With four applications of (2.7), we see that we can write $\varphi_{s}(\theta)$ in the form

$$
\varphi_{s}(\theta)=\frac{e^{s i \theta} H_{s}(\theta)}{\Gamma(\alpha+s) \Gamma(\beta-s) \Gamma(\gamma+s) \Gamma(\delta-s)},
$$

where

$$
H_{s}(\theta):={ }_{2} H_{2}\left[\begin{array}{cc}
1-\beta+s, & 1-\delta+s ; \\
\alpha+s, & \gamma+s ;
\end{array} e^{i \theta}\right] .
$$

Thus, we prove an analogue with $\varphi_{s}$ and $\varphi_{t}$ replaced by $H_{s}$ and $H_{t}$, respectively. At the end of our proof, we convert our result to (1.2).

For brevity, we introduce the notation

$$
\langle s\rangle_{n}:=\frac{(1-\beta+s)_{n}(1-\delta+s)_{n}}{(\alpha+s)_{n}(\gamma+s)_{n}} .
$$


In particular, we can then write

$$
H_{s}(\theta)={ }_{2} H_{2}\left[\begin{array}{cc}
1-\beta+s, & 1-\delta+s ; \\
\alpha+s, & \gamma+s ;
\end{array} e^{i \theta}\right]=\sum_{n=-\infty}^{\infty}\langle s\rangle_{n} e^{i n \theta} .
$$

Proof. By the quotient rule for derivatives,

$$
\frac{d}{d \theta}\left\{\frac{H_{s}(\theta)}{H_{t}(\theta)} e^{(s-t) i \theta}\right\}=\frac{\Delta}{e^{2 t i \theta} H_{t}^{2}(\theta)},
$$

where

$$
\Delta=e^{t i \theta} H_{t}(\theta) \frac{d}{d \theta}\left\{e^{s i \theta} H_{s}(\theta)\right\}-e^{s i \theta} H_{s}(\theta) \frac{d}{d \theta}\left\{e^{t i \theta} H_{t}(\theta)\right\} .
$$

Using the notation above and in the previous paragraph, and setting $k=m+n$ in the second equality below, we find that

$$
\begin{aligned}
\Delta & =i \sum_{m, n=-\infty}^{\infty}(s-t+n-m)\langle s\rangle_{n}\langle t\rangle_{m} e^{(s+t+n+m) i \theta} \\
& =i \sum_{k, n=-\infty}^{\infty}(s-t-k+2 n)\langle s\rangle_{n}\langle t\rangle_{k-n} e^{(s+t+k) i \theta} \\
& =i \sum_{k=-\infty}^{\infty}(s-t-k)\langle t\rangle_{k} e^{(s+t+k) i \theta} \sum_{n=-\infty}^{\infty} \frac{s-t-k+2 n}{s-t-k}\langle s\rangle_{n}\langle k+t\rangle_{-n} .
\end{aligned}
$$

Observe that the inner sum above is a well-poised ${ }_{5} H_{5}$, requiring that

$$
\operatorname{Re}(\alpha+\beta+\gamma+\delta)>3
$$

for convergence. Thus, we can use (2.3) to obtain the evaluation

$$
\begin{aligned}
& { }_{5} H_{5}\left[\begin{array}{rcccc}
1+\frac{1}{2}(s-t-k), & 1-\alpha-t-k, & 1-\beta+s, & 1-\gamma-t-k, & 1-\delta+s ; \\
\frac{1}{2}(s-t-k), & \alpha+s, & \beta-t-k, & \gamma+s, & \delta-t-k ;
\end{array}\right] \\
& =\Gamma\left[\begin{array}{c}
\alpha+t+k, \quad \gamma+t+k, \quad \beta-t-k, \quad \delta-t-k \\
1+s-t-k, 1-s+t+k, \alpha+\gamma+s+t+k-1, \beta+\delta-s-t-k-1
\end{array}\right]
\end{aligned}
$$

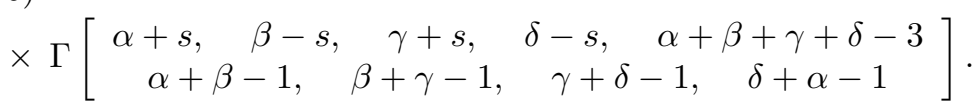

Using the evaluation (4.6) in (4.5) and simplifying the expressions involving gamma functions and rising factorials, we find that

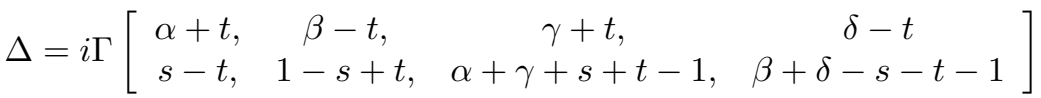

$$
\begin{aligned}
& \times \Gamma\left[\begin{array}{c}
\alpha+s, \beta-s, \gamma+s, \delta-s, \alpha+\beta+\gamma+\delta-3 \\
\alpha+\beta-1, \beta+\gamma-1, \gamma+\delta-1, \delta+\alpha-1
\end{array}\right] \\
& \times e^{i(s+t) \theta} \sum_{k=-\infty}^{\infty} \frac{(s+t-\beta-\delta+2)_{k}}{(s+t+\alpha+\gamma-1)_{k}} e^{i k \theta} \text {. }
\end{aligned}
$$


We next apply Horn's bilateral binomial theorem (2.5) with $a=s+t-\beta-\delta+2$ and $b=s+t+\alpha+\gamma-1$, subject to the condition $\operatorname{Re}(\alpha+\beta+\gamma+\delta)>4$. Thus,

$$
\sum_{k=-\infty}^{\infty} \frac{(s+t-\beta-\delta+2)_{k}}{(s+t+\alpha+\gamma-1)_{k}} e^{i k \theta}={ }_{1} H_{1}\left[\begin{array}{l}
s+t-\beta-\delta+2 ; \\
s+t+\alpha+\gamma-1 ;
\end{array} e^{i \theta}\right]
$$

$$
=\left(-e^{i \theta}\right)^{2-\alpha-\gamma-s-t}\left(1-e^{i \theta}\right)^{\alpha+\beta+\gamma+\delta-4} \frac{\Gamma(\alpha+\gamma+s+t-1) \Gamma(\beta+\delta-s-t-1)}{\Gamma(\alpha+\beta+\gamma+\delta-3)} .
$$

Now substitute (4.8) into (4.7), use (2.7), and cancel common gamma function factors to arrive at

$$
\begin{aligned}
\Delta= & e^{(s+t) i \theta}\left(-e^{i \theta}\right)^{2-\alpha-\gamma-s-t}\left(1-e^{i \theta}\right)^{\alpha+\beta+\gamma+\delta-4} \\
& \times i \Gamma\left[\begin{array}{c}
\alpha+s, \beta-s, \gamma+s, \delta-s, \alpha+t, \beta-t, \gamma+t, \delta-t \\
s-t, 1-s+t, \alpha+\beta-1, \beta+\gamma-1, \gamma+\delta-1, \delta+\alpha-1
\end{array}\right] \\
= & \frac{i}{\pi} \sin \{\pi(s-t)\}\left(2 \sin \frac{\theta}{2}\right)^{\alpha+\beta+\gamma+\delta-4} e^{i(\pi-\theta)(\alpha-\beta+\gamma-\delta+2 s+2 t) / 2} \\
& \times \Gamma\left[\begin{array}{c}
\alpha+s, \beta-s, \gamma+s, \delta-s, \alpha+t, \beta-t, \gamma+t, \delta-t \\
\alpha+\beta-1, \beta+\gamma-1, \gamma+\delta-1, \delta+\alpha-1
\end{array}\right] .
\end{aligned}
$$

Lastly, substituting (4.9) into (4.3) and then reformulating the result according to the relation (4.1) between $\varphi_{t}(\theta)$ and $H_{t}(\theta)$, we derive the identity

$$
\frac{d}{d \theta}\left\{\frac{\varphi_{s}(\theta)}{\varphi_{t}(\theta)}\right\}=\frac{i \sin \{\pi(s-t)\}\left(2 \sin \frac{\theta}{2}\right)^{\alpha+\beta+\gamma+\delta-4} e^{i(\pi-\theta)(\alpha-\beta+\gamma-\delta+2 s+2 t) / 2}}{\pi \varphi_{t}^{2}(\theta) \Gamma(\alpha+\beta-1) \Gamma(\beta+\gamma-1) \Gamma(\gamma+\delta-1) \Gamma(\delta+\alpha-1)}
$$

which is (1.3). The proof is thus complete.

Let $\phi_{t}(\theta)=e^{-t i \theta} \varphi_{t}(\theta)$. We end our paper with Ramanujan's rendition of Entry 1.2. given by

$$
\begin{aligned}
& \frac{d}{d \theta}\left\{\frac{\phi_{s}(\theta)}{\phi_{t}(\theta)}\right\} \\
& \quad=\frac{i \sin \{\pi(s-t)\}\left|2 \sin \frac{\theta}{2}\right|^{\alpha+\beta+\gamma+\delta-4} e^{i(\alpha-\beta+\gamma-\delta+2 s-2 t)\{(\pi-\theta) / 2+\pi[\theta /(2 \pi)]\}}}{\pi \phi_{t}^{2}(\theta) \Gamma(\alpha+\beta-1) \Gamma(\beta+\gamma-1) \Gamma(\gamma+\delta-1) \Gamma(\delta+\alpha-1)} .
\end{aligned}
$$

Note that Ramanujan's function $\phi_{s}(\theta)$ does not have the factor $e^{s i \theta}$ in $\varphi_{s}(\theta)$, defined in (1.2). The second major difference between the two formulas is in the exponent of $e$ on the right-hand sides. One would guess that $[x]$ in Ramanujan's exponent denotes the greatest integer less than or equal to $x$. The powers of $2 \sin \left(\frac{1}{2} \theta\right)$ in both (1.3) and (4.10) are the same, except that Ramanujan has absolute values around $2 \sin \left(\frac{1}{2} \theta\right)$. In conclusion, except for multiplicative expressions of absolute value equal to 1 , the other parts of formulas (4.10) and (1.3) are identical.

\section{REFERENCES}

[1] G. E. Andrews, R. Askey, and R. Roy, Special Functions, Cambridge University Press, Cambridge, 2000. MR 1688958(2000g:33001)

[2] J. Dougall, On Vandermonde's theorem and some more general expansions, Proc. Edinburgh Math. Soc. 25 (1907), 114-132.

[3] M. E. Horn, Bilateral binomial theorem, SIAM Problem 03-001 (2003). 
[4] S. Ramanujan, The Lost Notebook and Other Unpublished Papers, Narosa, New Delhi, 1988. MR0947735 (89j:01078)

[5] L. J. Slater, Generalized Hypergeometric Functions, Cambridge University Press, Cambridge, 1966. MR0201688 (34:1570)

Department of Mathematics, University of Illinois, 1409 West Green Street, UrBANA, ILLINOIS 61801

E-mail address: berndt@math.uiuc.edu

Dipartimento di Matematica, Università degli Studi di Lecce, Lecce-Arnesano P. O. Box 193, 73100 Lecce, Italy

E-mail address: chu.wenchang@unile.it 